\title{
Cultura y tecnología: distribuciones humanas
}

\author{
Eva AlADRo Vico \\ Universidad Complutense de Madrid \\ ealadro@ccinf.ucm.es
}

Recibido: $11 / 07 / 2012$

Aceptado: 23/10/2012

\section{Resumen}

Este texto revisa la relación entre las innovaciones tecnológicas, costumbres y hábitos y la cultura de nuestras sociedades, mostrando su articulación. De la revisión de los aspectos culturales, tecnológicos y sociales del uso de herramientas y la constitución de los hábitos se deduce toda una perspectiva nueva que muestra la cultura como un sistema de externalización y ampliación de las capacidades humanas ejemplo es el lenguaje-, y a la vez, la tecnología como un sistema que permite internalizar y hacer propios artefactos y medios que ensanchan la capacidad humana de comunicar.

Palabras Clave:tecnología, hábitos, herramientas, límites humanos, cultura.

\section{Culture and technology: human distributions}

\begin{abstract}
This text reflects upon the relationship between technologies, habits and culture, deepening into their articulation. Reviewing the cultural, technological and social aspects of tools and habits, it shows a new perspective about how culture externalizes and makes wider the possibilities of human improvement language is an example-, while technologies allow the internalization of objects and tools that make broader the human ability to communicate.
\end{abstract}

Key words:technology, habits, tools, culture, borders of human development.

\section{Referencia normalizada}

ALADRO VICO, Eva (2012): "Cultura y tecnología: distribuciones humanas". Estudios sobre el mensaje periodístico. Vol. 18, núm. especial octubre, págs.: 35-43. Madrid, Servicio de Publicaciones de la Universidad Complutense.

Sumario: 1. Introducción y metodología: los hábitos, contenidos convertidos en herramientas. 2. Desarrollo; 2.1 Los hábitos y la tecnología: herramientas que se convierten en sujetos y contenidos; 2.2 Mentes extendidas y acción sin fronteras; 2.3 La cultura y los hábitos: extensiones y adquisiciones. 3. Conclusiones; 3.1 Un paso más: oficio y arte, la simbiosis entre el infinito y la materia. 4. Referencias bibliográficas

\section{Introducción y metodología: los hábitos, contenidos convertidos en herra- mientas}

Alva Noé afirma que un hábito es "un contenido convertido en herramienta" (Noé, 2010: 145). Cuando la mente humana automatiza una conducta, disminuye la consciencia de ella, para favorecer la acción inmediata. Nuestra consciencia, como la psicología cognitiva mostró, automatiza imágenes y conductas y les concede tal autonomía que llegamos a considerarlas ajenas a nosotros.

Nuestra mente tiene una plasticidad absoluta para redibujar sus límites y para asumir o ceder entornos, tecnologías o esquemas corporales. Este artículo aborda, reflexivamente, este fenómeno y sus consecuencias. Noé estudia cómo la mente humana genera sistemas simbióticos desde la infancia, bien con otros individuos -el sistema madre-bebé es uno- bien con objetos o tecnologías, en los que la mente reorganiza su propio sistema básico de propiocepción y movimiento añadiendo o eliminando - au- 
tomatizando- elementos. Este autor señala que somos seres expandidos, extendidos y dinámicos (Noé, 2010: pág. 93) capaces de sustituir unos sentidos por otros, y disponer del entorno en relaciones de sistema.

Parte de la capacidad de habituación humana es una reducción de carga cognitiva. Cuando generamos un hábito, el cerebro no consciente se hace cargo de desarrollar las proyecciones, programaciones y reacciones, dejando a la mente consciente liberada del control de esas acciones. El llamado saber pericial supone prescindir de la consciencia de reglas, normas o elementos constituyentes, es más amplio que el pensamiento, y usa la economía y la relajación (Noé, 2010: 132).

La fortaleza con la que se instalan hábitos es en mucha medida resultado de nuestra mente empática y de las denominadas neuronas espejo (Rizzolatti y Sinigaglia, 2006). Ellas almacenan la información motora y perceptiva automática en nuestra mente, con finalidades de elaborar habilidades y aprendizajes. La empatía de las neuronas espejo es una automatización básica. El cerebro humano puede asimilar, y adoptar automáticamente, muchas reacciones. Así interviene también el cerebro emocional de la amígdala.

Goleman (1996) ha analizado cómo la instauración de conductas automáticas emocionales por reflejo ante determinados estímulos es un mecanismo básico del sistema de autodefensa humano. Diversas escuelas que analizan las programaciones de las conductas (PNL, AT, TC) muestran cómo una sola experiencia puede cristalizar en un complejo hábito de comportamiento. Así se trata, por ejemplo, el control de las depresiones (Burns, 1980; Beck 1989).

Un ejemplo sorprendente de la capacidad de nuestro sistema de habituación y automatización es el fenómeno que Ramachandran (1998), neuropsicólogo hindú, calificó como "terapia de espejo" para el tratamiento del llamado síndrome de la mano fantasma. Este síndrome ilustra la capacidad de automatización mental de los hábitos, que genera percepciones falsas. El síndrome del miembro fantasma hace que personas con un miembro amputado sigan sintiéndolo, e incluso, que dolores en el mismo tengan difícil tratamiento por tratarse de un fallo en el sistema.

Ramachandran generó un dispositivo mediante el cual una percepción ficticia generada con un espejo, del miembro amputado, permite a la mente tratarlo con técnicas de relajación y fisioterapia. Lo que nos llama la atención es cómo en este caso, la habituación que generaba una coordinación sensible ficticia entre un brazo y el cerebro, puede ser utilizada en su base automática para desconectar un hábito establecido, como si introdujéramos un nuevo paso en el programa cerebral psicomotor mediante la inserción de una imagen falsa, que la mente básica motora asume como auténtica, retomando con ella el hábito instrumental original.

La terapia del espejo es un ejemplo de una realidad absoluta en la mente humana: los hábitos, que automatizan nuestras conductas y convierten en herramientas desconectadas de la mente consciente los contenidos adquiridos, son un modo como economizamos atención y dedicación cerebral, y de algún modo, reducimos y distribuimos la carga de capacidades y actividades que debemos realizar con la mente consciente. Los hábitos saltan los límites infranqueables de las condiciones corporales, generan impresiones autónomas y hacen fácil la superación de esas condiciones, 
permitiendo también como estudiamos en este ensayo, colectivizar sus contenidos y con ello, traspasarlos a los demás.

\section{Desarrollo}

\subsection{Los hábitos y la tecnología: herramientas que se convierten en sujetos y con- tenidos}

La capacidad de convertir contenidos en hábitos, y con ello, utilizarlos sin cargar cognitivamente con ellos, explica la riqueza y complejidad de nuestra mente. Muchas de las conductas que llevamos a cabo a diario están automatizadas en hábitos y han desaparecido de nuestra atención consciente: habilidades psicomotoras, manejo de tecnologías, aprendizaje de habilidades corporales, uso de lenguajes, y de protocolos de acción social y cultural. Todos estos "conocimientos" que entraron en nuestras vidas de diversas maneras, han pasado al control inconsciente generado por el hábito, y se disparan. Silenciosamente, estos hábitos producen acciones y efectos sensoriales, emocionales, actitudinales, culturales, difícilmente detectables precisamente por su carácter reactivo.

Bruno Latour (2001) nos da el contrapunto a la frase de Alva Noé con la que iniciábamos este texto, al afirmar cómo las herramientas y objetos no humanos pueden vertebrarse como agentes de plena capacidad en nuestros sistemas culturales, como son el conocimiento científico, la cultura o la vida social, una vez que pasan a ser "automáticos". Define Latour las herramientas como "la extensión de las habilidades sociales a los no humanos" (Latour, 2001: 252). Si los hábitos automatizan, instrumentan y finalmente desagregan de nuestra consciencia elementos semánticos, para permitir su uso automático, también somos capaces de absorber y otorgar consciencia y significado, por así decir, a instrumentos y herramientas separados de nuestra mente, pero que se vinculan a ésta y convierten en prótesis simbólicas, según la expresión de Derrick De Kerckhove (1999a). Es decir: si un hábito es un contenido convertido en herramienta, una herramienta es un contenido que un hábito convierte en interno humano.

La capacidad de simbiosis con el entorno, generada por esa habilidad mental, es una vieja conocida de los estudios de la Escuela de Toronto a la que pertenece De Kerckhove. McLuhan y McLuhan (2009) hablaron de la ley de la extensión sensorial de todo medio, según la cual somos capaces de generar una prolongación o proyección sensorial de algún sentido o facultad a partir de la interacción con elementos del entorno. La capacidad de prótesis sensorial, extendiendo nuestras facultades y sentidos, tiene sus leyes de reversión, como la que genera una atrofia o pérdida de sensibilidad inducida por una facultad o sentido excesivos: recordemos el célebre ejemplo del bastón del ciego de Diderot, donde el sentido del tacto del ciego en la punta de su bastón exige la anulación de su capacidad perceptiva en los dedos. De manera similar, otros mecanismos proyectivos y extensivos de comunicación generan atrofias o redistribuciones de cargas sensoriales, como estudia la Mediología.

En las Leyes de los medios, los McLuhan (2009) analizaron las tecnologías como extensiones de contenidos - de sentidos, facultades, representaciones y conocimientosa las que la habituación nos permite acceder cuando nos es preciso, pero al mismo 
tiempo, mantienen su carácter diverso y autónomo al individuo. Podemos llegar a la simbiosis con las tecnologías porque nuestra mente puede extenderse y delegar en soportes externos parte de sus acciones habituales. La capacidad del hábito, que construye esquemas más allá de los límites naturales del cuerpo, según necesidades y usos, cumple este cometido.

Toda la tecnología de comunicaciones actual muestra la capacidad de simbiosis y de nuevos modelos de cognición intermedia, como los llama De Kerckhove (1999a: 47). La mente se extiende en múltiples sistemas de almacenamiento de información -de los que hablaremos ahora-, y se distribuye para una mayor eficacia en sistemas tecnológicos de manipulación e instrumentación - del ordenador al robot.

La siempre presente habilidad humana para internalizar instrumentos y herramientas culturales se ha disparado de tal manera, que De Kerckhove describe campos unificados de estructuras humanas y tecnológicas orgánicamente relacionadas, en las que la tecnología empieza a ser más abundante que el sujeto humano al que se acopla, de modo que se hace real que la mente se extiende y, como si pudiera dársele la vuelta como a un guante, desarrolla su dinamismo interior en el exterior, ampliando y extendiendo en proyecciones culturales todos esos elementos (De Kerckhove, 1999b).

Latour estudia los procesos culturales que objetivan, independizan y generan sistemas en los que los sujetos interactúan con vínculos, instrumentos, contenidos conceptuales al igual que lo hacen con otros humanos o con las herramientas tecnológicas. Las redes de intermediarios van creando relaciones que involucran a un gran número de elementos generando sistemas sólidos de dinamismo, como es la propia ciencia (Latour 2001: 120). La capacidad de conectarse a redes y de que éstas adquieran capacidades por encima de los límites del pensamiento o del cuerpo individual es la clave de muchas construcciones sociales, como estudiaremos luego. No es extraño que los constructos lógicos o los conceptos, el lenguaje, mismo, se conviertan en actores.

\subsection{Mentes extendidas y acción sin fronteras}

La habituación a una herramienta externa, como puede ser un ordenador, o el teléfono móvil, genera un sistema solidario en el que la mente se extiende, tal y como Clarck y Chalmers (2011) explican continuando el debate filosófico- cognitivo del internalismo y externalismo de la mente humana. Otros autores llaman a este proceso mente "distribuida" (vid. Salomon, 1992). Clarck y Chalmers describen nuestra mente capaz de depositar capacidades como la memoria o el razonamiento lógico en tecnologías ensambladas a nuestras actividades, y se puede generar más eficacia y rapidez mental cuando se interactúa con estas prótesis - por ejemplo, la velocidad de reacción puede ser más rápida si en ella interviene la psicomotricidad asociada a la manipulación de un mando a distancia, que si se produce únicamente en el mundo mental imaginario de un individuo- (Clarck y Chalmers, 2011: 16 y ss). Pensar ensamblados a una tecnología puede ser más rápido que pensar a secas.

Clarck y Chalmers hacen hincapié en cómo el sistema ensamblado que genera una tecnología y un individuo, supera las barreras planteadas por las condiciones del entorno o psicobiológicas, generando una "co-evolución". La capacidad de desprenderse de procesos motores simples en la automatización por hábito, y la capacidad para in- 
tegrar elementos externos en una habituación que los convierte en propios, es la clave de los procesos coevolutivos.

Los sistemas de evolución en los que la extensión de la mente a una herramienta externa, con la que se ha generado un sistema, termina por desarrollar nuevas capacidades en el individuo sometido al sistema ensamblado, son la clave para subir un nivel en las reflexiones que estamos describiendo. El ejemplo que ponen Chalmers y Clark (2010: 19 y ss.) es el del dispositivo nadador del pez, según el cual, este animal es capaz de generar en el agua las adecuadas corrientes y volutas que, a su vez, constituyen apoyos -tecnologías-, sobre los que el animal genera su movimiento. Pero, a su vez, la natación del pez ya ha desarrollado músculos adecuados al sistema que genera él mismo en las corrientes.

No puedo olvidar la historia de los cangrejos Heike que Carl Sagan contaba en su serie "Cosmos". Esos cangrejos del mar de Japón son resultado de una curiosa interacción, preservados por los pescadores debido al parecido de su caparazón con el rostro de los guerreros samurái. El mito, la memoria de un suceso terrible, la casualidad y la acción y contacto humanos han llegado a hacer co-evolucionar de tal manera a estos cangrejos que su abundancia y su carácter único -seleccionados artificialmente en un sistema humano cultural- acentúa y resalta el sorprendente parecido entre sus cuerpos y los rostros humanos japoneses. Este ensamblaje es un símbolo de la extensión y maleabilidad de las formas y especies a partir de su interacción.

\subsection{La cultura y los hábitos: extensiones y adquisiciones}

Las extensiones generadas por los seres humanos para ampliar sus capacidades y facultades terminan por repercutir en ampliaciones de los propios elementos básicos que las generaron. Cuando se crean en la cultura sistemas de ensamblaje aparece la coevolución, es decir, surgen capacidades nuevas, que dejan atrás los límites del desarrollo que un ser aislado puede tener.

Bruno Latour y antes que él, fenomenólogos sociales como Schutz o Luckman advirtieron que las construcciones mentales también se convierten en elementos externos, objetivados, herramientas transformables y adaptables en cada sistema social y cultural. Latour (2001) ha analizado estas interacciones mostrando cómo el contacto con objetos o elementos no humanos conforma agentes en sistemas, y la relación establecida imprime su forma a cuantos participan en ella.

Lo que nos encontramos, si unimos los dos planos de Latour y Alva Noé, es que nuestra mente puede extenderse y también contraerse en contacto con herramientas, se distribuye instrumentalizando sus propias funciones y automatizándolas en los hábitos, economiza su atención mediante esta operación, pero también, amplía sus propias capacidades absorbiendo herramientas a las que naturaliza. Si dichas herramientas son constructos culturales de enorme valor, contenidos desarrollados en contacto con muy profundas fuentes de sentido, que por este procedimiento han sido legados a la cultura, este sistema es la puerta de acceso a un desarrollo ilimitado.

Las fronteras de la mente humana, ante estos fenómenos, se nos presentan fluidas e indefinidas, tanto por su borde exterior como por su lado interno. Los hábitos nos permiten expulsar de la consciencia elementos y facultades cuya automatización re- 
sulta mucho mejor para el ser humano: por ejemplo, la habituación semántica a significados de los signos nos permite trabajar con la comunicación mediante los lenguajes. Por el lado externo, la absorción empática de prótesis generadas desde el exterior -tecnologías, códigos, cajas de herramientas culturales y sociales- nos permite amplificar nuestra capacidad de experiencia, hasta límites muy por encima de nuestras posibilidades individuales.

Putnam (1990) fue uno de los primeros filósofos en reflexionar sobre la división del trabajo lingüístico en la vida social. En su teoría, el lenguaje es uno de los componentes instrumentales que, externalizados en el mundo cultural, en un grado u otro, el individuo acopla a su mente en un proceso de adquisición que le permitirá, por su completa adecuación, extender infinitamente sus capacidades de pensamiento y de comunicación.

El lenguaje constituye efectivamente una caja de herramientas cuyo desarrollo y conservación ha sido desligado de la capacidad individual para pasar a ser parte del trabajo social dividido y organizado en la cultura. Pero, a su vez el lenguaje es también una tecnología protésica cuyo ensamblaje con la mente humana tiene lugar de un modo tan automático e instintivo que Chomsky (1999) pudo afirmar con total acierto que su estructura profunda, capaz de generar las infinitas creaciones de la expresión verbal, estaba en contacto con el fondo universal del ser humano.

Cómo es posible que una "caja de herramientas" a la que se accede culturalmente, se integre de tal modo en la profunda mente humana que genere la expresión creativa individual de un modo único, sigue siendo un misterio para la lingüística y la filosofía. Pero lo cierto es que el uso del lenguaje, adquirido e innato a la vez, es resultado de la indefinición de los límites de la mente humana, que puede depositar en soportes externos -la sociedad, la academia, la tradición cultural- elementos creados por ella como es el lenguaje, y después incorporarlos y extenderse nuevamente en ellos como ocurre en la creación, innovación y expresión individual.

El lenguaje es un ejemplo muy claro de la capacidad de absorción de una extensión automatizada que se reincorpora en cada individuo. La cultura es un sistema de extensiones que almacenan y atesoran capacidades de expresión y acción humana, que se ha conseguido materializar y distribuir en la vida social por los procesos de hábito. Dichas extensiones y prótesis son, a su vez, adaptables e incorporables a cada individuo, pudiéndose llegar a una automatización total en su uso.

Esta perspectiva acaba con el debate del relativismo y universalismo culturales que tanto han preocupado a pensadores de los últimos lustros. La cultura es resultado de un sistema de distribución y traspaso de hallazgos y de legados de experiencias humanas. Puede automatizarse y desprenderse de cada individuo, e instituirse en distribuciones que permanecen en el mundo social. Ello es posible gracias a los procesos mentales de generación de hábitos y automatismos que externalizan contenidos y capacidades. Pero también, la cultura es absorbida e internalizada como una herramienta acoplable de tal manera que la mente humana se extiende y evoluciona con ella. Ello es posible gracias a la capacidad mental de simbiosis y de extensión.

Como un cuerpo desmontable y recomponible, que adquiriera capacidad orgánica una vez que es reensamblado, la cultura permite que la comunicación traslade capacidades y dinamismos rompiendo los límites individuales y circunstanciales. 
Hábito, externalización, distribución, extensión, empatía y automatización son partes de un ciclo en el que la cultura está implicada profundamente. Son capacidades que generan un sistema de comunicación que rompe las barreras del ser individual, y de la capacidad individual, para vincular al entorno cada ser. La comunicación es el proceso que borra las fronteras entre el individuo y el entorno, generando sistemas de adaptación que son bien conocidos para la psicología evolutiva de hace un siglo (Piaget, 1985). Esos sistemas son la prueba de que la mente humana no discierne, a este nivel de eficacia, entre lo interno y lo adquirido, entre lo propio y lo ajeno, igual entre lo creado por ella misma y lo aprehendido por influencia exterior.

\section{Conclusiones}

\subsection{Un paso más: oficio y arte, la simbiosis entre el infinito y la materia}

Ya que hemos contrapuesto las citas de Noé y Latour para avanzar en nuestra disquisición, terminaremos avanzando un paso de gigante, contraponiendo a dos autores de enorme profundidad en la reflexión sobre cultura. La filósofa Simone Weil (1995: 174) reflexionó sobre el trabajo manual y en cierta ocasión recordaba cómo un dicho popular afirmaba que una herida causada en las manos de un artesano por la herramienta de su oficio "hacía entrar" en la sangre de dicho artesano el oficio en cuestión.

Todo trabajo humano supone una simbiosis con una herramienta que puede ensamblarse en un sistema que tiene un carácter único, evoluciona unido, y resulta equilibrado, útil y automático. Weil conocía la capacidad reflejante que la naturaleza y sus objetos tienen para permitir que el ser humano sienta la seguridad necesaria para avanzar en la creación y el trabajo. La capacidad de co-evolución con el elemento externo al hombre, está expresada en esa sabiduría.

La herramienta artesana, como la materia sobre la que el artista trabaja, son soportes de una relación que supera las barreras del individuo humano para establecer una unidad expresiva que va más allá de la mente, y ensambla la naturaleza del medio, del entorno, y la del artista o artesano. John Berger (2012) muy recientemente nos recordaba que todo arte es un diálogo entre el artista y la materia con la que trabaja. En ese diálogo afirma Berger lo que muchos aristas reconocen: las formas vienen proporcionadas desde "dentro" de la roca, la materia con la que el hombre trabaja, y éste se limita, dice Berger, a "sacarlas".

Los sistemas del arte y del trabajo artesano popular nos muestran la simbiosis entre el individuo y su entorno para la expresión de la existencia. Esa simbiosis ha barrido literalmente los contornos entre lo que es un individuo, lo que es humano, y lo que es suprahumano, un sistema formado por el entorno, la materia, la naturaleza y el hombre.

Otro filósofo de quien Weil aprendió mucho, llamado René Guénon, reflexionaba a comienzos del XX sobre la mutilación que supone la maquinización del trabajo manual (Guénon, 1997: 58 y ss). Ante la llegada de un mundo industrial de terrible fuerza, en el que se rompía esa relación de mutuo apoyo que describía Simone Weil, las capacidades de simbiosis con ese entorno y herramientas no crecían, sino que mermaban. En lugar de extensiones, aquellos medios se convirtieron en narcóticos, en atrofiantes. 
Guénon y Simone Weil ilustraron en múltiples imágenes y símbolos cómo la unión con la herramienta y la materia permite superar los planos de dificultad para llegar más allá de lo individual, hasta lo que no está definido todavía. En las reflexiones de Weil en torno a los trabajos no serviles, y en las de Guénon sobre la tradición de los oficios y su valor primordial, hallamos la base para pensar que toda herramienta que se asume debe pasar a ser la causa de una mejora, y cómo las capacidades humanas se potencian más allá de sí mismas cuando se limitan con la dedicación del oficio.

Cuando una tecnología se hace propia y entra en la sangre del individuo, o cuando un hábito consigue extraer de la profundidad de la mente humana algo que puede manipularse y trasladarse como un útil, tiene lugar una operación de unión entre la potencia y el acto, entre la virtualidad y la realidad. En toda la sabiduría ancestral se conoce esa capacidad creativa que se da cuando no media el pensamiento y las acciones del creador son rápidas y veloces, sin espacio entre la intención y la acción. Es lo que los artistas milenarios japoneses llamaban "muga", cuando no hay separación entre el pensamiento y la acción.

Las tecnologías actuales nos vuelven a mostrar la ausencia de límites en el desarrollo humano. Hoy en día más aún vemos volver a las máquinas al viejo uso lábil en el que los hombres pueden superar con ellas las limitaciones y redibujar su propia potencialidad. Uniéndose con las herramientas, automatizando su uso, las personas hacemos efectiva la superación de nuestras limitaciones y asumimos en los actos creativos la infinitud que se ofrece ante nosotros para desarrollar.

\section{Referencias bibliográficas}

BECK, Aaron (1989): Love is Never Enough. New York, Harper and Row.

BERGER, John (2012): Sobre el dibujo. Barcelona, Gustavo Gili.

BURNS, David (1980): Feeling Good. New York, Avon Books.

CHOMSKY, Noam, (1999): Aspectos de una teoría de la sintaxis. Barcelona, Gedisa.

CLARCK, Andy, y CHALMERS, David J. (2011): La mente extendida. Revista CIC, Cuadernos de Información y Comunicación, número 16, pp.15-28.

DE KERCKHOVE, Derrick (1999a): La piel de la cultura. Barcelona, Gedisa.

DE KERCKHOVE, Derrick (1999b): Inteligencias en conexión. Barcelona, Gedisa.

GOLEMAN, Daniel (1996): Inteligencia emocional. Barcelona, Kairós.

GUÉNON, René (1997): El reino de la cantidad y los signos de los tiempos. Barcelona, Paidós.

LATOUR, Bruno, (2001): La esperanza de Pandora. Barcelona, Gedisa.

MCLUHAN. Marshall y MCLUHAN, Eric,(2009): Las leyes de los medios. Revista CIC, Cuadernos de Información y Comunicación, número 14, 285-316.

NOÉ, Alva (2010): Fuera de la cabeza. Barcelona, Kairós.

PIAGET, Jean (1985): El nacimiento de la inteligencia en el niño. Barcelona, Crítica. 
PUTNAM, Hilary (1990): Representación y realidad: Un balance crítico de funcionalismo. Barcelona, Gedisa.

RIZZOLATTI, Giacomo y SINIGAGLIA, Corrado (2006): Las neuronas espejo Los mecanismos de la empatía emociuonal. Barcelona, Paidós Ibérica.

RAMACHANDRAN, Vilayanur S. (1998): Phantoms in the Brain. New York, William Morrow.

SALOMON, Gavriel, PERKINS, David N., y GLOBERSON, Tamar (1992): “Coparticipando en el conocimiento: la ampliación de la inteligencia humana con las tecnologías inteligentes". Comunicación, lenguaje y educación, 13, pp. 6-22

WEIL, Simone (1995): La gravedad y la gracia. Madrid, Trotta.

\section{Eva ALADRO VICO}

Profesora Titular

Departamento de Periodismo III

Facultad de Ciencias de la Información

Universidad Complutense de Madrid

ealadro@ccinf.ucm.es 